\title{
Phase transitions in KDP crystals with the complex organic and inorganic impurities
}

\author{
B.Strukov, I.Shnaidshtein, S.Grabovsky \\ Lomonosov Moscow State University, Moscow, 119992, Russia
}

Received September 4, 2006

\begin{abstract}
In this paper we present the results of the study of dielectric and thermal properties of KDP crystals doped with different complex organic and inorganic molecules which decorate different (pyramidal or prismatic) crystal growth sectors. It is shown that the properties of stained and transparent parts of the crystal in comparison with the corresponding parts of the nominally pure crystal are different for KDP crystals grown by means of traditional (slow) and rapid growth technique. The difference of the domain contribution into dielectric constant of the polar phase and its characteristic hysteresis in pure and dyed crystals, transition temperature position, form of the specific heat anomaly are presented and analyzed. The most probable model of incorporation of the complex impurity molecules into KDP structure is proposed.
\end{abstract}

Key words: ferroelectrics, impurities, dielectric constant, dye molecules, specific heat, crystal growth

PACS: $81.10 . A j, 61.72 . W w, 77.84 . F a, 77.80 . D j, 77.80 . B h$

\section{Introduction}

It is well known that $\mathrm{KH}_{2} \mathrm{PO}_{4}(\mathrm{KDP})$ crystals represent the most typical hydrogen-bonded ferroelectric with the order-disorder phase transition. This model crystal was studied more in detail than other ferroelectrics and is the most convenient object for the construction of macroand microscopic theories of such transitions [1,2]. Besides, KDP crystals have outstanding nonlinear optical properties; for some applications in laser optics, very large plates of the crystal were claimed [3], therefore the methods of rapid growth of KDP-type crystals were successfully developed $[3,4]$. At paraelectric phase KDP crystals belong to the point group $\overline{4} 2 m$ and are faceted by the sets of $\{101\}$ (pyramidal) and $\{100\}$ (prismatic) faces. For the crystal grown using the traditional technique with low supersaturation, the growth rate is $0.5-1 \mathrm{~mm} /$ day. Here the crystal grows only in the [001] direction with the quite slow growth of the $\{100\}$ faces due to the blocking effect of polyvalent ion impurities in solution on prismatic faces [5]. On the other hand, the crystals grown with the rate exceeding $10 \mathrm{~mm} /$ day (rapid growth technique [3,4]) start from the point seed and grow uniformly for both prismatic and pyramidal faces since in this case the blocking effect of inevitable solution impurities becomes much smaller and is distributed more uniformly on all faces. Surprisingly, optical and nonlinear optical properties of the crystals grown using the rapid growth technique turn out to be of the same quality as the crystals grown using a traditional method. It should be noted that the difference in the properties of pyramidal and prismatic sectors of KDP crystals grown by means of the traditional technique was detected in some experimental works. For example, the domain pattern is quite different in different sectors revealing the regular stripes which are parallel to (100) or (010) plane in the pyramidal sector, whereas in the prismatic sectors the domains width and length are distributed irregularly and are completely restored after repeatedly passing through Curie temperature [6]. Therefore, only the samples originated from the pyramidal sectors were considered in the number of works.

Thus, it becomes evident that it is important to identify the "pure" KDP samples obtained from certain growth sectors before introducing the impurities into the crystal. It is known that the anomalously high dielectric constant in ferroelectric phase in KDP crystals is a result of a higher mobility of the domain walls which is extremely sensitive to the presence of different kinds 
of defects [7-9]. In what follows we choose the domain contribution into dielectric constant in the polar phase as a parameter closely related to the impurity density and defect concentration in the sample.

The effect of organic dye molecules of hematein in KDP crystal has been originally observed in [10]. The authors revealed for the first time that this dye can recognize the different faces of growing KDP crystal staining only every other prism face. They found out that the transition temperature in the doped sectors was depressed by $2 \mathrm{~K}$. Recently it was shown that many other different dyes and complex inorganic molecules can be introduced in the KDP crystal lattice selectively in pyramidal or prismatic sectors $[11,12]$ but no information about thermodynamic properties of the stained and nonstained parts has been reported. The puzzling peculiarity of the case is the lack of similarity between guest molecules and host crystal structure; in this sense the dye impurities can be considered as "nonisomorphic" to the crystal lattice and still there is a problem in understanding the capability of the growing crystal to accommodate such "inconvenient" inclusions violating the principles of isomorphism and discriminating between different faces. It seems evident that these partly ordered "inclusions" into piezoelectric inorganic matrix have to develop the local strains and accompanying local polarization. Therefore, it is possible to create the "strong" polar defects in ferroelectric crystal and to study their effect on the phase transition. In this paper we present a short review of our studies of the complex organic and inorganic molecules concerning dielectric and thermal properties of KDP crystals. The dye staining pyramidal or prismatic sectors of the host crystal are considered. The difference in the properties of the "pure" KDP crystals grown by means of traditional and rapid growth techniques is considered as a necessary background for the separation of defects - induced effects [13].

\section{Experimental}

The following crystals grown using rapid growth technique were in our disposal: pure KDP, traditionally and rapidly grown; KDP + organic dye Amaranth (A), KDP + organic dye Chicago Sky Blue (CSB) - both dyes stain only pyramidal sectors; KDP + organic dye Xylenol Orange (XO), KDP + inorganic dye Pento-Chloro-Ruthenate (PCR) - both dyes stain only prismatic sectors. Impurity distributions are represented schematically in figure 1. Spectral analysis revealed the average concentration of dye molecules as 1-5 molecules of dye at $10^{5}$ molecules of KDP, average volume of crystals was about several tens of $\mathrm{cm}^{3}$. All samples were cut perpendicular to the polar $c$ axe as plates with the size about $5 \times 8 \times 1 \mathrm{~mm}^{3}$. The thin layers of silver paste was used as electrodes. For dielectric measurements the automatic capacitance bridge with the typical frequency of $10 \mathrm{kHz}$ was used. The rate of the sample cooling/heating far from $T_{\mathrm{C}}$ was $0.5-1 \mathrm{~K} / \mathrm{min}$ and $0.1-0.2 \mathrm{~K} / \mathrm{min}$ in the vicinity of the transition point. Specific heat data were obtained using a computerized adiabatic calorimeter in the temperature range $4-160 \mathrm{~K}$. In this case the sample has a form of cylinder with a diameter $8 \mathrm{~mm}$ and height $13 \mathrm{~mm}$. The temperature step was $2 \mathrm{~K}$ far from $T_{\mathrm{C}}$ and $0.02 \mathrm{~K}$ in the vicinity of the transition point. The accuracy of the specific heat measurements was $0.5 \%$ and was checked by comparing our data with the classical work [14] at low temperatures.

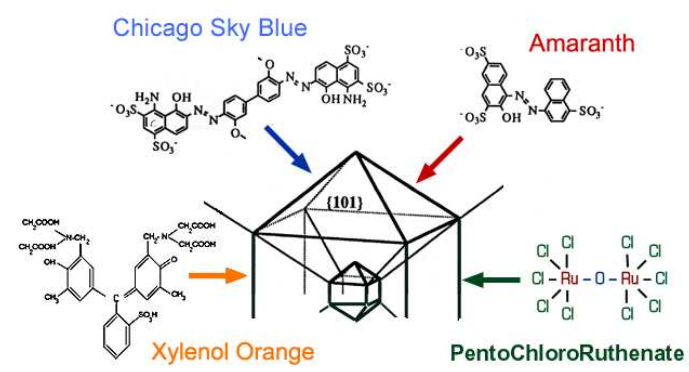

Figure 1. Schematic presentation of dye molecules incorporated into different growth sectors of KDP crystal. 


\section{Dielectric properties: pyramid-stained crystals}

As it was pointed in Introduction (and it will be necessary for the interpretation of the further results) the initial habitus of the nominally pure KDP crystals is connected with the presence of polyvalent ions $\left(\mathrm{Cr}^{+3}, \mathrm{Fe}^{+3}, \mathrm{Al}^{+3}, \mathrm{Sn}^{+4}\right)$ in a solution. It is generally accepted that these impurities inhibit the growth of the crystal in the [001] direction because they are easily adsorbed by the prismatic faces and block their growth if the crystal is grown by traditional method from the slightly supersaturated solution. At the same time, the growth of the pyramidal faces is not prevented by these impurities. On the other hand, the rapid growth technique makes it possible to overcome the blocking effect of impurities and to grow the crystal uniformly so that the final size of the crystal almost does not depend on the initial size of the seed. It is clearly seen in figure 2 that the temperature dependencies of dielectric constant of KDP crystal grown by both methods is quite different for different sectors: curve 1 shows the classical picture for the pyramidal sector of traditionally grown crystal: there is a temperature region in the polar phase where $\varepsilon$ has an anomalously high values ("plateau" region); near $90 \mathrm{~K} \varepsilon$ sharply decreases, revealing the "freezing out" of the contribution of domain walls mobility. It is evident from curves 2,3 that both the pyramidal and prismatic sectors of the rapidly grown crystal have the following features in comparison with the previous case: the lower value of $\varepsilon$ at $T_{\mathrm{C}}$ and a suppressed domain mobility in polar phase. If we accept that the domain mobility contribution to dielectric constant can be

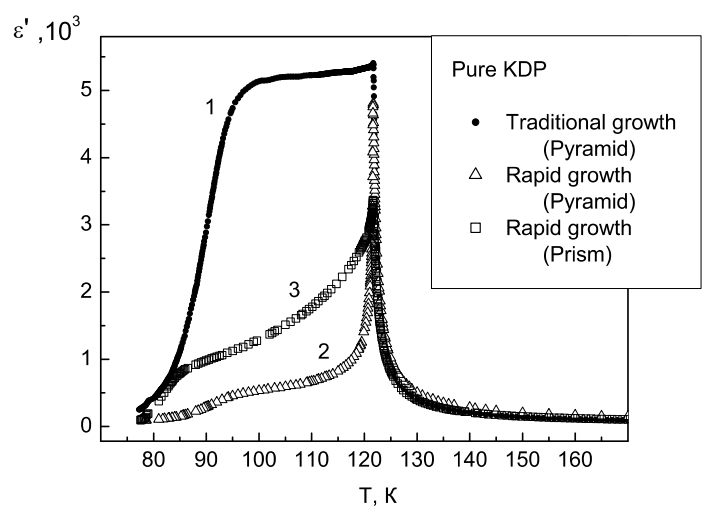

Figure 2. Temperature dependencies of dielectric constant for the nominally pure KDP crystals grown using different methods: 1-pyramid (traditional method), 2-pyramid (rapid growth), 3prism (rapid growth).
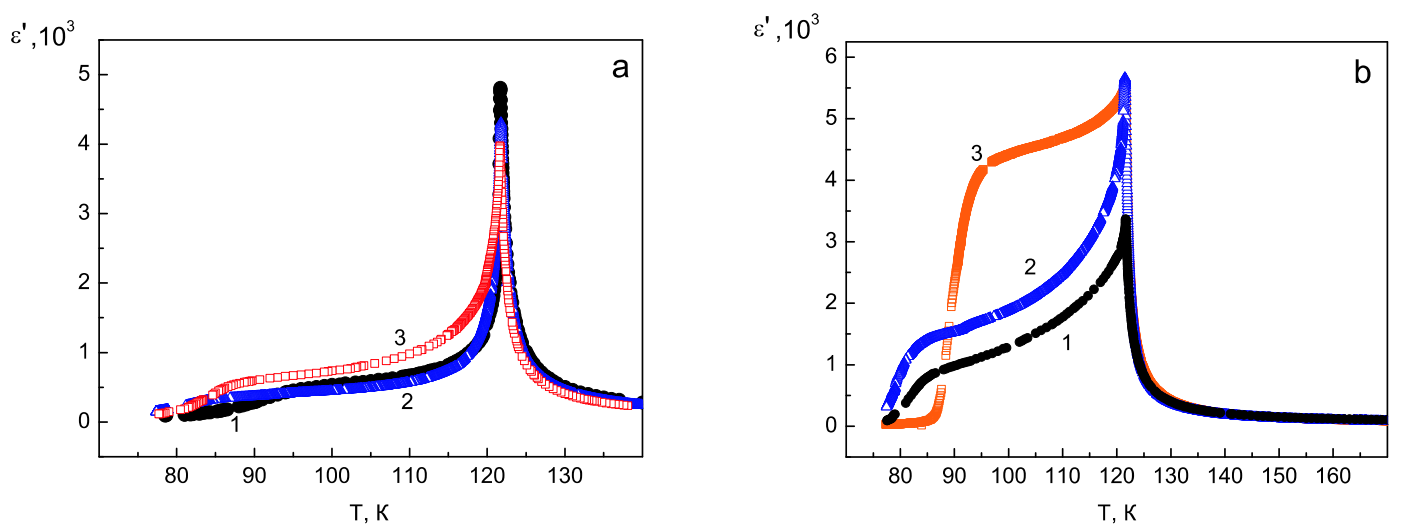

Figure 3. Temperature dependence of dielectric constant of pure $\operatorname{KDP}(1)$, KDP+CSB (2), and $\mathrm{KDP}+\mathrm{A}(3)$ crystals. (a) - pyramidal sector, (b) - prismatic sector.

taken as a measure degree of the crystal quality, one can conclude from figure 2 that the pyramidal sector in rapidly grown KDP crystal in one case is more defected than the prismatic one and that 
the kinetics of the crystal growth determines the impurity distribution between the growth sectors of the crystal.

Temperature dependence of dielectric constant of pure KDP crystals and KDP crystals with decorated pyramidal sectors $(\mathrm{KDP}+\mathrm{CSB}$ and $\mathrm{KDP}+\mathrm{A})$ is presented in figure 3 . All crystals were grown using a rapid growth technique. Data for both dyed (pyramidal) and transparent (prismatic) sectors were inspected. It is seen that the pyramidal sectors of pure and decorated crystals have got very similar temperature dependencies of dielectric constant; at the same time there is a striking difference between these dependencies for the transparent prismatic sectors. It is clear from figure 3 that the prismatic sector of KDP+A crystal contains much less "blocking" polyvalent impurities than KDP $+\mathrm{CSB}$ crystal. As a result, the prism of KDP $+\mathrm{A}$ crystal becomes almost as free from these impurities as it is in pure KDP crystal grown by the classical method. It means that the molecules of organic dyes A being introduced into KDP, staining the pyramidal sectors of the crystal and leaving prismatic sectors unstained, practically do not affect the degree of defectiveness of a pyramid and are responsible for a substantial purification of the prismatic sector from the blocking polyvalent ions and for the reappearance of the "plateau", typical of the high quality KDP crystals grown using the traditional method. This purification can be connected with the formation of stable complexes of organic dye molecules with polyvalent cation background impurities and neutralization of their effect upon the prismatic sectors.

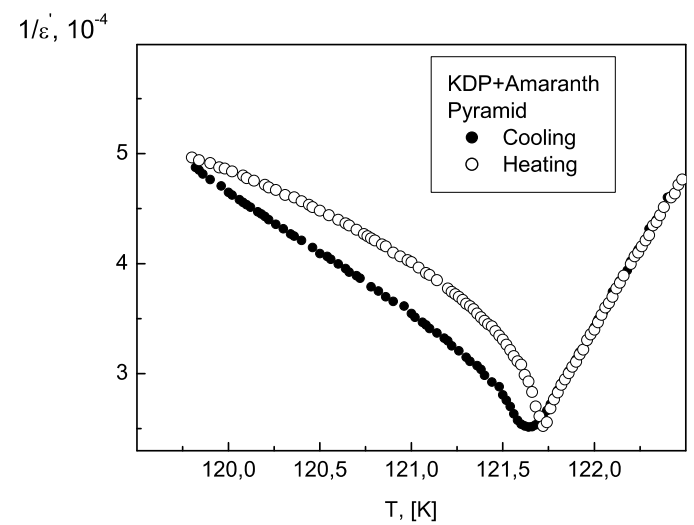

Figure 4. Temperature dependence of the inverse dielectric constant of pyramidal sector of $\mathrm{KDP}+\mathrm{A}$ near $T_{\mathrm{C}}$.

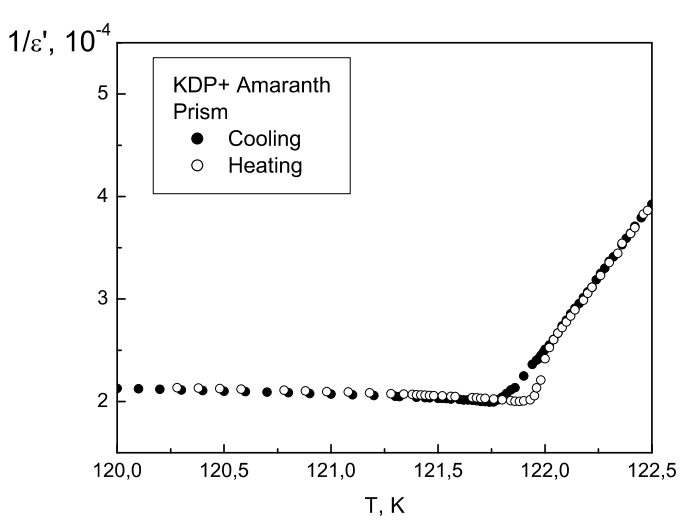

Figure 5. Temperature dependence of the inverse dielectric constant of prismatic sector of $\mathrm{KDP}+\mathrm{A}$ near $T_{\mathrm{C}}$.

Measurements of dielectric constant in the vicinity of the transition point $\left(T_{\mathrm{C}}=121.8 \mathrm{~K}\right)$ revealed two peculiarities of the doped samples. The first one is a small (near $0.1 \mathrm{~K}$ ) hysteresis of the transition point on cooling and heating which is typical for the first-order phase transition close to the tricritical point. The second is the remarkable difference of dielectric constant on cooling and heating in ferroelectric phase for the pigmented pyramidal sectors (figure 4). The latter effect is not pronounced for the transparent (prismatic) sectors (figure 5). Moreover, in KDP $+\mathrm{A}$ crystal the form of $\varepsilon(T)$ dependence near $T_{\mathrm{C}}$ looks (for the prism sector) almost completely similar to the one for the pure KDP crystal grown using the traditional method [13]. The small smearing of the phase transition (which is more pronounced for KDP + CSB crystal) without a visible shift of the transition temperature is evident for the stained parts of the crystal.

\section{Thermal properties: pyramid-stained crystals}

We have measured the specific heat $C(T)$ for the prism and pyramid of pure KDP, KDP $+\mathrm{CSB}$ and $\mathrm{KDP}+\mathrm{A}$. It was found that far from $T_{\mathrm{C}}$, all experimental curves are practically identical within an accuracy of measurements. In the region of phase transition $\left(T_{\mathrm{C}} \pm 0.3 \mathrm{~K}\right)$ the situation in pure and doped crystals was revealed as quite different. The sharp decrease of maximum value of the 
specific heat at $T_{\mathrm{C}}$ and the change of the form of $C(T)$ dependencies were observed for the stained pyramidal parts of $\mathrm{KDP}+\mathrm{CSB}$ and $\mathrm{KDP}+\mathrm{A}$ crystal (figure 6a); for the relatively pure transparent prismatic sectors of KDP+CSB crystal the reproducible splitting of the specific heat anomaly was detected (figure 6b).
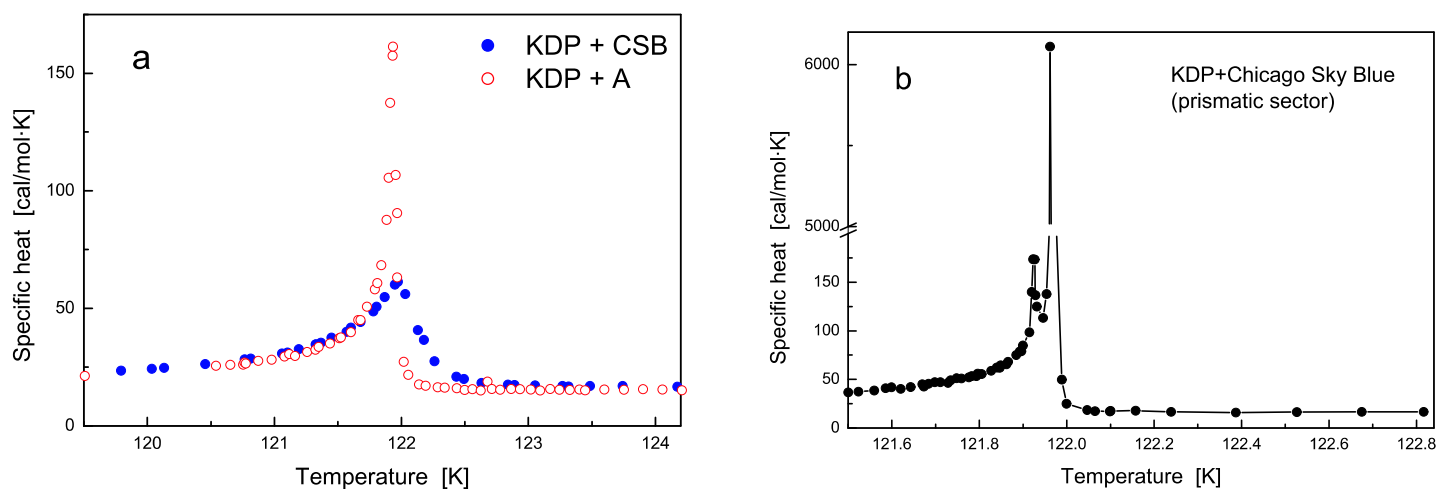

Figure 6. Temperature dependence of specific heat near $T_{\mathrm{C}}$ for the stained pyramidal parts of $\mathrm{KDP}+\mathrm{CSB}$ and KDP+A crystals (a); the same for the prismatic part of crystal KDP+CSB (b).

It is seen that the form of $C(T)$ dependencies in figure 6 a looks like the one for pure KDP crystal located in the external electric field $[15,16]$ with the only difference that the maximum value of the specific heat of pigmented parts of the crystals is not shifted to higher temperatures. This phenomenon can be explained within the theory of the phase transitions in defected crystals predicted the displacement of the transition point to lower temperatures [17]. To analyse the reason of splitting of the specific heat anomaly observed in relatively pure prismatic sector of KDP $+\mathrm{CSB}$ (figure $6 \mathrm{~b}$ ) we should take into account the fact that such a phenomenon was observed earlier for some defected KDP crystals $[18,19]$. In our case we can assume that even in the transparent part of the crystal there is some amount of the CSB dye molecules - polar defects - which are randomly distributed in the crystal forming the inner biasing field. It was demonstrated by Duckek and Falk in [20] that for the first-order ferroelectric phase transition which is close to a tricritical point, the occurrence of metastable states is possible in an external electric field. That is the case when

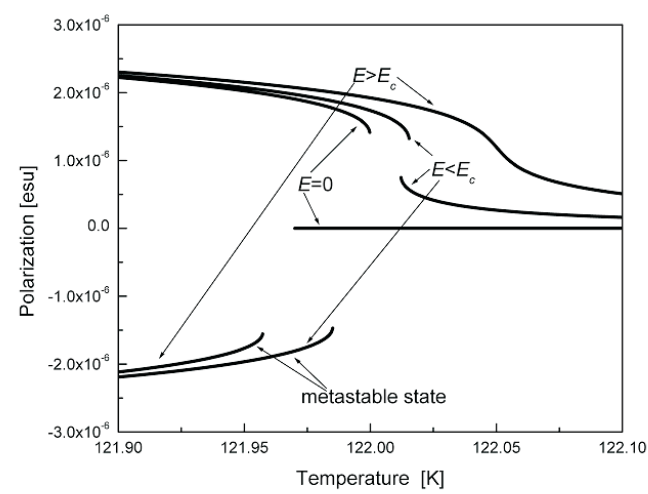

Figure 7. Temperature dependence of polarization for KDP crystal in different external electric field. Upper curves - for the stable states $(E \uparrow \uparrow P)$, lower - for the unstable states $(E \uparrow \downarrow P)$. $E_{\mathrm{c}}-$ electrical "critical" field removing the jump of polarization at $T_{\mathrm{C}}$.

polarization of the ferroelectric is directed opposite to the field. For the inner biasing field created by the defects themselves the situation is possible when one part of the crystal volume will be in the stable state (polarization $P_{+}$is along the field) whereas the other part will be in metastable 
state (polarization $P_{-}$is counter the field). This idea is supported by the fact that the spontaneous polarization calculated from the specific heat data was found to be by $8-10 \%$ less in doped crystal in comparison with the pure one.

Denoting the latter (relative) part as $s$, we shall have for the anomalous part of specific heat for such a crystal as $C=(1-s) C\left(P_{+}\right)+s C\left(P_{-}\right)$. The part of $P(T)$ dependencies constructed according to Duckek and Falk theory is presented in figure 7 with the Landau parameters taken from [16]; the corresponding specific heat temperature dependence is shown in figure $6 \mathrm{~b}$ as a solid line $(s=0.04)$; the coincidence of the experimental points with the calculated curve is fairly good.

\section{Dielectric properties: prism-stained crystals}

It has been already pointed out that dyes can selectively penetrate into different sectors of the crystal. This feature of dyes is probably connected with the stereochemical affinity of the dye with the crystal lattice [11]. The nature of this affinity is not clear at present although some assumption for the dyes with the relatively simple structure will be done in the next section. The question is whether the properties of the doped KDP crystal with the stained prism are similar to the above considered case of stained pyramid. In the only paper where the phase transition in the prism-stained KDP crystal was studied (with hematein as a dopant) the authors revealed the noticeable lowering of the transition point [10]; we have not detected this effect for the pyramidstained crystals. Dielectric data for two prism-stained crystal $-\mathrm{KDP}+\mathrm{PCR}$ and KDP+XO were obtained. The small size of the samples gave us no chance to measure specific heat of these crystals. The structural formulas for the both molecules - Pento-Cloro-Ruthenate and Xelenol Orange are presented in figure 1. As to PCR, the evident stereochemical affinity of the matrix and impurity causes the joining of the molecules to prismatic sector; evidently, the anion $\left[\mathrm{Ru}_{2} \mathrm{Cl}_{10} \mathrm{O}\right]^{4-}$ formed in a solution penetrates as a whole into the growing crystal. The structure of the organic dye has no visible features of affinity with the crystal lattice and this impurity is completely nonisomorphic to the matrix; it is not clear at present why these molecules "choose" mainly prismatic sectors.
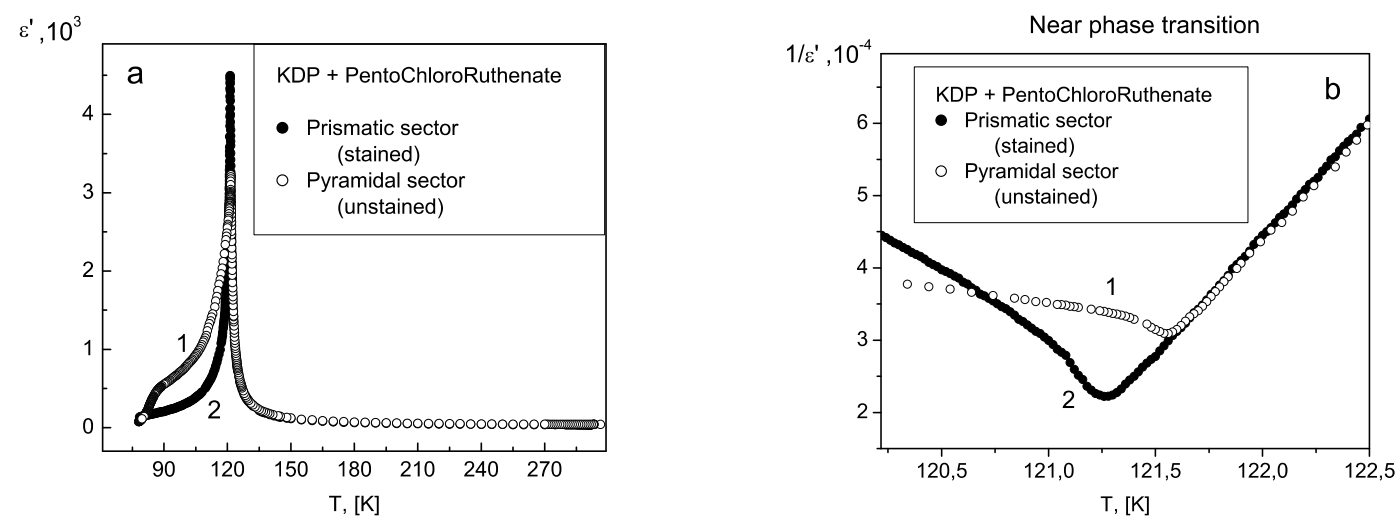

Figure 8. Temperature dependence of dielectric constant of KDP $+\mathrm{PCR}$ in the wide temperature range (a) (1-transparent pyramid, 2 - pigmented prism) and of the reciprocal dielectric constant in the vicinity of $T_{\mathrm{C}}(\mathrm{b})(1.2-$ the same as in (a)).

The temperature dependence of dielectric constant of KDP+PCR crystal is shown in figure 8a. It is evident that there is almost complete suppressing of the domain contribution into dielectric constant of the stained prismatic sector, and $\varepsilon(T)$ anomaly has a symmetrical form unusual for KDP crystals with the Curie-Weiss law being fulfilled for both phases. For the transparent pyramidal sector $\varepsilon(T)$ dependence is qualitatively identical with the one for the pure KDP crystal showing the typical "shoulder" in the polar phase. It is remarkable that the data in the very vicinity of the transition point reveal the difference of transition temperatures for samples cut from different sectors of the doped crystal (figure 8b): the maximum value of dielectric constant corresponds to 
$121.60 \mathrm{~K}$ for the transparent pyramidal sector and $121.25 \mathrm{~K}$ for the stained prisma. Therefore, our data are in accordance with the results of [10] obtained for KDP crystals doped by hematein.

In the case of $\mathrm{KDP}+\mathrm{XO}$ crystal, the results were less determined. The comparison of its properties with the properties of pure crystal shows that the impurity penetrates to both prismatic and pyramidal sectors, though its concentration in the pyramid is much less than in the prism; the shift of the transition point was not detected, but the suppressing of the domain contribution in dielectric constant was found to be remarkable in the more intensively stained pyramidal sector.

\section{The possible model of penetrating: Amaranth case}

The problem of the large complex dye molecules penetrating into KDP structure was discussed in $[11,12]$. In accordance with the proposed model the stereochemical affinity is supposed to be an important factor of the partially ordered disposition of the impurity molecules in the crystal matrix. Thus, the end groups $\mathrm{SO}_{3}^{-}$of the dye molecules can presumably occupy the positions of $\mathrm{PO}_{4}^{-}$groups in KDP lattice. If one considers a dye molecule as a rigid complex, the strong local mechanical deformation of crystal lattice can be proposed around this molecule. So far as we found no data on the interatomic distances for the dye molecule Amaranth possessing the simplest structure, we used the quantum-chemical set of program GAMESS [21] for the calculation of the space geometry of this molecule exploiting the principle of the minimum energy. The direct comparison of the parameters of the dye molecule with the crystal structure of KDP crystal revealed the following features. The most probable region of the impurity molecule inclusion is the diagonal plane of the elementary cell (figure 9). The mechanical strain accompanying such a disposition can be considered as the increasing of $a_{1}$ parameter by $0.55 \AA$ and simultaneous decreasing of $a_{2}$ by $0.56 \AA$ in the initial nonstrained cell plus shear in the basic plane, decreasing the angle between $a_{1}$ and $a_{2}$ by $6 \%$. It

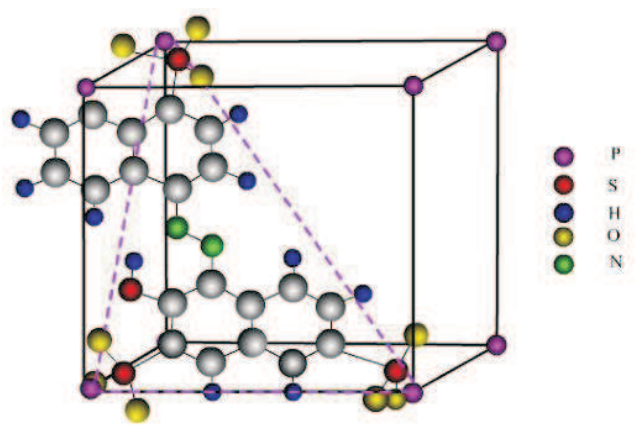

Figure 9. Presumable scheme of the disposition of Amaranth molecule in KDP structure. means that the local strains in the region of the molecule localization are similar to the strains of KDP lattice arising at the phase transition. Of course, such speculations become very difficult for the dyes with the more intricate molecular structure.

\section{Conclusions}

We have studied dielectric and thermal properties of KDP crystals doped with dye molecules staining different sectors of growth of crystal matrix. The dielectric measurements revealed that the dye molecules pigmenting the pyramidal sectors and leaving the prismatic ones transparent, only slightly affect the domain contribution into dielectric constant of the polar phase and do not shift the transition point; at the same time, they are responsible for cleaning the prismatic sectors from polyvalent metal impurities. This causes a remarkable increase of dielectric constant of the prism and the appearance of the "plateau" region which is typical of the high quality KDP crystals. On the contrary, the complex impurities selectively entering the prismatic sector almost completely suppress the domain contribution into dielectric constant of this sector and slightly decrease the transition temperature, leaving the dielectric constant of the transparent pyramidal sector unchanged. It is shown that the specific heat of the pure and doped KDP is different in the interval of about $1 \mathrm{~K}$ near $T_{\mathrm{C}}$ for the pyramid-stained crystals. The appearance of the biasing field directed counter the spontaneous polarization was considered in interpreting the obtained specific heat temperature dependencies. The presumable model of Amaranth dye molecule entering the KDP lattice is proposed. 


\title{
8. Acknowledgements
}

Authors thank the RFBR for the financial support of this research (project 05-02-16873a), N. Zaitseva and L. Carman for the samples of KDP $+\mathrm{CSB}$ and KDP+A crystals, B. Kahr for the samples of KDP+PCR crystals and I. Pritula and Yu. Velikhov for the samples of KDP+XO crystals. The discussions with L. Rashkocich are very much appreciated.

\section{References}

1. Mitsui T., Tatsuzaki I., Nakamura E., An Introduction to the Physics of Ferroelectrics. Gordon and Breach, New York, 1976.

2. Vaks V.G., Zein N.E, Strukov B.A., Phys. Stat. Sol. (a), 1975, 30, 801.

3. Zaitseva N., Carman L., Progress in Crystal Growth and Characterization of Materials, 2001, 43, 1.

4. Rashkovich L.N., Vestn. Akad. Nauk SSSR, 1984, 9, 15 (in Russian).

5. Rashkovich L.N., KDP Family of Crystals. Hilger-Bristol, London, 1991.

6. Abe K., J. Phys. Soc. Jap., 1987, 56, 757.

7. Nakamura E., Ferroelectrics, 1992, 135, 237.

8. Kamysheva L.N., Burdanina N.A., Zhukov O.K., Izvestia Akad. Nauk SSSR, seria fiz., 1970, 34, 2612 (in Russian).

9. Sidorkin A.S., Domain Structure in Ferroelectrics and Related Materials. Nauka, Moscow, 2000 (in Russian).

10. Blathner H., Matthias B., Merz B., Helv. Phys. Acta, 1946, 19, 415.

11. Subramony J.A., Jang S.-H., Kahr B., Ferroelectrics, 1997, 191, 293.

12. Kahr B., Gurney R.W., Chem. Rev., 2001, 101, 893.

13. Grabovsky S.V., Shnaidshtein I.V., Strukov B.A., Kristallografia, 2003, 48, 357 (in Russian).

14. Stephenson C.C., Hooley J.G., Journ. Am. Chem. Soc., 1944, 66, 1397.

15. Strukov B.A., Amin M.S., Kopchik V.A., Phys. Stat. Sol., 1968, 27, 741.

16. Sandvold E., Fossheim K., J. Phys. C, 1986, 19, 1481.

17. Levanyuk A.P., Sigov A.S., Defects and Structural Phase Transitions. Gordon and Breach, New York, 1988.

18. Pompe G, Hegenbarth E., Phys. Sol. State, 1970, 12, 455.

19. Sandvold E., Laegreid T., Fossheim K., Fossum J.O., Phase Transitions, 1988, 11, 145.

20. Dukek G., Falk G., Z. Phys., 1970, 240, 93.

21. Schmidt M.W., Baldridge K.K., Boatz J.A., J. Comput. Chem., 1993, 14, 1347.

\section{Фазові переходи в кристалах KDP з комплексними органічними та неорганічними домішками}

\author{
Б.Струков, І.Шнадштейн, С.Грабовський \\ Московський державний університет ім. Ломоносова, 119992 Москва, Росія \\ Отримано 4 вересня 2006 р.
}

У цій статті ми представляємо результати вивчення діелектричних та термічних властивостей кристалів KDP, легованих різноманітними комплексними органічними та неорганічними молекулами, які покривають різні (пірамідальні або призматичні) ділянки росту кристалу. Показано, що властивості зафарбованих і прозорих частин кристалу у порівнянні з відповідними ділянками номінально чистого кристалу є різними для кристалів KDP, вирощених з використанням традиційної (повільної) та швидкої технологій вирощування. Представлено та проаналізовано відмінність доменного вкладу у діелектричну сталу полярної фази та характерний гістерезис для чистих і зафарбованих кристалів, розташування температури переходу та форму аномалії теплоємності. Запропоновано найбільш правдоподібну модель включення молекул комплексних домішок у структуру KDP.

Ключові слова: сегнетоелектрики, домішки, діелектична стала, молекули фарбника, теплоємність, вирощування кристалів

PACS: $81.10 . A j, 61.72 . W w, 77.84 . F a, 77.80 . D j, 77.80 . B h$ 